\title{
Combinatorial interpretations of the Jacobi-Stirling numbers
}

\author{
Yoann Gelineau and Jiang Zeng \\ Université de Lyon, Université Lyon 1, \\ Institut Camille Jordan, UMR 5208 du CNRS, \\ F-69622, Villeurbanne Cedex, France \\ gelineau@math.univ-lyon1.fr, zeng@math.univ-lyon1.fr
}

Submitted: Sep 24, 2009; Accepted: May 4, 2010; Published: May 14, 2010

Mathematics Subject Classifications: 05A05, 05A15, 33C45; 05A10, 05A18, 34B24

\begin{abstract}
The Jacobi-Stirling numbers of the first and second kinds were introduced in the spectral theory and are polynomial refinements of the Legendre-Stirling numbers. Andrews and Littlejohn have recently given a combinatorial interpretation for the second kind of the latter numbers. Noticing that these numbers are very similar to the classical central factorial numbers, we give combinatorial interpretations for the Jacobi-Stirling numbers of both kinds, which provide a unified treatment of the combinatorial theories for the two previous sequences and also for the Stirling numbers of both kinds.
\end{abstract}

\section{Introduction}

It is well known that Jacobi polynomials $P_{n}^{(\alpha, \beta)}(t)$ satisfy the classical second-order Jacobi differential equation:

$$
\left(1-t^{2}\right) y^{\prime \prime}(t)+(\beta-\alpha-(\alpha+\beta+2) t) y^{\prime}(t)+n(n+\alpha+\beta+1) y(t)=0 .
$$

Let $\ell_{\alpha, \beta}[y](t)$ be the Jacobi differential operator:

$$
\ell_{\alpha, \beta}[y](t)=\frac{1}{(1-t)^{\alpha}(1+t)^{\beta}}\left(-(1-t)^{\alpha+1}(1+t)^{\beta+1} y^{\prime}(t)\right)^{\prime}
$$

Then, equation (1.1) is equivalent to say that $y=P_{n}^{(\alpha, \beta)}(t)$ is a solution of

$$
\ell_{\alpha, \beta}[y](t)=n(n+\alpha+\beta+1) y(t)
$$


Table 1: The first values of $\operatorname{JS}_{n}^{k}(z)$

\begin{tabular}{c|cccccc}
$k \backslash n$ & 1 & 2 & 3 & 4 & 5 & 6 \\
\hline 1 & 1 & $z+1$ & $(z+1)^{2}$ & $(z+1)^{3}$ & $(z+1)^{4}$ & $(z+1)^{5}$ \\
2 & & 1 & $5+3 z$ & $21+24 z+7 z^{2}$ & $85+141 z+79 z^{2}+15 z^{3}$ & $341+738 z+604 z^{2}+222 z^{3}+31 z^{4}$ \\
3 & & & 1 & $14+6 z$ & $147+120 z+25 z^{2}$ & $1408+1662 z+664 z^{2}+90 z^{3}$ \\
4 & & & & 1 & $30+10 z$ & $627+400 z+65 z^{2}$ \\
5 & & & & & & $55+15 z$ \\
6 & & & & & &
\end{tabular}

In [5, Theorem 4.2], for each $n \in \mathbb{N}$, Everitt et al. gave the following expansion of the $n$-th composite power of $\ell_{\alpha, \beta}$ :

$$
(1-t)^{\alpha}(1+t)^{\beta} \ell_{\alpha, \beta}^{n}[y](t)=\sum_{k=0}^{n}(-1)^{k}\left(P^{(\alpha, \beta)} S_{n}^{k}(1-t)^{\alpha+k}(1+t)^{\beta+k} y^{(k)}(t)\right)^{(k)}
$$

where $P^{(\alpha, \beta)} S_{n}^{k}$ are called the Jacobi-Stirling numbers of the second kind. They [5, (4.4)] also gave an explicit summation formula for $P^{(\alpha, \beta)} S_{n}^{k}$ numbers, showing that these numbers depend only on one parameter $z=\alpha+\beta+1$. So we can define the Jacobi-Stirling numbers as the connection coefficients in the following equation:

$$
x^{n}=\sum_{k=0}^{n} \mathrm{JS}_{n}^{k}(z) \prod_{i=0}^{k-1}(x-i(z+i)),
$$

where $\mathrm{JS}_{n}^{k}(z)=P^{(\alpha, \beta)} S_{n}^{k}$, while the Jacobi-Stirling numbers of the first kind can be defined by inversing the above equation:

$$
\prod_{i=0}^{n-1}(x-i(z+i))=\sum_{k=0}^{n} \operatorname{js}_{n}^{k}(z) x^{k}
$$

where $\mathrm{js}_{n}^{k}(z)=P^{(\alpha, \beta)} s_{n}^{k}$ in the notations of [5].

It follows from (1.2) and (1.3) that the Jacobi-Stirling numbers $\operatorname{JS}_{n}^{k}(z)$ and $\mathrm{js}_{n}^{k}(z)$ satisfy, respectively, the following recurrence relations:

$$
\left\{\begin{array}{l}
\mathrm{JS}_{0}^{0}(z)=1, \quad \mathrm{JS}_{n}^{k}(z)=0, \quad \text { if } k \notin\{1, \ldots, n\} \\
\operatorname{JS}_{n}^{k}(z)=\mathrm{JS}_{n-1}^{k-1}(z)+k(k+z) \mathrm{JS}_{n-1}^{k}(z), \quad n, k \geqslant 1 .
\end{array}\right.
$$

and

$$
\left\{\begin{array}{l}
\mathrm{js}_{0}^{0}(z)=1, \quad \mathrm{js}_{n}^{k}(z)=0, \quad \text { if } k \notin\{1, \ldots, n\}, \\
\mathrm{js}_{n}^{k}(z)=\mathrm{js}_{n-1}^{k-1}(z)-(n-1)(n-1+z) \mathrm{js}_{n-1}^{k}(z), \quad n, k \geqslant 1 .
\end{array}\right.
$$

The first values of $\operatorname{JS}_{n}^{k}(z)$ and $\mathrm{js}_{n}^{k}(z)$ are given, respectively, in Tables 1 and 2 .

As remarked in $[4,5,1]$, the previous definitions are reminiscent to the well-known Stirling numbers of the second (resp. the first) kind $S(n, k)$ (resp. $s(n, k))$, which are 
Table 2: The first values of $j s_{n}^{k}(z)$

\begin{tabular}{c|ccccc}
$k \backslash n$ & 1 & 2 & 3 & 4 & 5 \\
\hline 1 & 1 & $-z-1$ & $2 z^{2}+6 z+4$ & $-6 z^{3}-36 z^{2}-66 z-36$ & $24 z^{4}+240 z^{3}+840 z^{2}+1200 z+576$ \\
2 & & 1 & $-3 z-5$ & $11 z^{2}+48 z+49$ & $-50 z^{3}-404 z^{2}-1030 z-820$ \\
3 & & & 1 & $-6 z-14$ & $35 z^{2}+200 z+273$ \\
4 & & & & 1 & $-10 z-30$ \\
5 & & & &
\end{tabular}

defined (see [2]) by

$$
x^{n}=\sum_{k=0}^{n} S(n, k) \prod_{i=0}^{k-1}(x-i), \quad \prod_{i=0}^{n-1}(x-i)=\sum_{k=0}^{n} s(n, k) x^{k} .
$$

and satisfy the following recurrences:

$$
\begin{array}{llrl}
S(n, k) & =S(n-1, k-1)+k S(n-1, k), & & n, k \geqslant 1, \\
s(n, k) & =s(n-1, k-1)-(n-1) s(n-1, k), & & n, k \geqslant 1 .
\end{array}
$$

The starting point of this paper is the observation that the central factorial numbers of the second (resp. the first) kind $T(n, k)$ (resp. $t(n, k)$ ) seem to be more appropriate for comparison. Indeed, these numbers are defined in Riordan's book [8, p. 213-217] by

$$
x^{n}=\sum_{k=0}^{n} T(n, k) x \prod_{i=1}^{k-1}\left(x+\frac{k}{2}-i\right),
$$

and

$$
x \prod_{i=1}^{n-1}\left(x+\frac{n}{2}-i\right)=\sum_{k=0}^{n} t(n, k) x^{k} .
$$

Therefore, if we denote the central factorial numbers of even indices by $U(n, k)=T(2 n, 2 k)$ and $u(n, k)=t(2 n, 2 k)$, then :

$$
\begin{aligned}
U(n, k) & =U(n-1, k-1)+k^{2} U(n-1, k), \\
u(n, k) & =u(n-1, k-1)-(n-1)^{2} u(n-1, k) .
\end{aligned}
$$

From (1.4)-(1.11), we easily derive the following result.

Theorem 1. Let $n, k$ be positive integers with $n \geqslant k$. The Jacobi-Stirling numbers $\mathrm{JS}_{n}^{k}(z)$ and $(-1)^{n-k} \mathrm{js}_{n}^{k}(z)$ are polynomials in $z$ of degree $n-k$ with positive integer coefficients. Moreover, if

$$
\begin{aligned}
\mathrm{JS}_{n}^{k}(z) & =a_{n, k}^{(0)}+a_{n, k}^{(1)} z+\cdots+a_{n, k}^{(n-k)} z^{n-k}, \\
(-1)^{n-k} \mathrm{js}_{n}^{k}(z) & =b_{n, k}^{(0)}+b_{n, k}^{(1)} z+\cdots+b_{n, k}^{(n-k)} z^{n-k},
\end{aligned}
$$

then

$$
a_{n, k}^{(n-k)}=S(n, k), \quad a_{n, k}^{(0)}=U(n, k), \quad b_{n, k}^{(n-k)}=|s(n, k)|, \quad b_{n, k}^{(0)}=|u(n, k)| .
$$


Note that when $z=1$, the Jacobi-Stirling numbers reduce to the Legendre-Stirling numbers of the first and the second kinds [4]:

$$
\mathrm{LS}(n, k)=\mathrm{JS}_{n}^{k}(1), \quad \operatorname{ls}(n, k)=\mathrm{js}_{n}^{k}(1)
$$

The integral nature of the involved coefficients in the above polynomials ask for combinatorial interpretations. Indeed, it is folklore (see [2]) that the Stirling number $S(n, k)$ (resp. $|s(n, k)|$ ) counts the number of partitions (resp. permutations) of $[n]:=\{1, \ldots, n\}$ into $k$ blocks (resp. cycles). In 1974, in his study of Genocchi numbers, Dumont [3] discovered the first combinatorial interpretation for the central factorial number $U(n, k)$ in terms of ordered pairs of supdiagonal quasi-permutations of $[n]$ (cf. $\S 2$ ). Recently, Andrews and Littlejohn [1] interpreted $\mathrm{JS}_{n}^{k}(1)$ in terms of set partitions (cf. $\S 2$ ).

Several questions arise naturally in the light of the above known results:

- First of all, what is the combinatorial refinement of Andrews and Littlejohn's model which gives the combinatorial counterpart for the coefficient $a_{n, k}^{(i)}$ ?

- Secondly, is there any connection between the model of Dumont and that of Andrews and Littlejohn?

- Thirdly, is there any combinatorial interpretation for the coefficient $b_{n, k}^{(i)}$ in the Jacobi-Stirling numbers of the first kind, generalizing that for the Stirling number $|s(n, k)|$ ?

The aim of this paper is to settle all of these questions. Additional results of the same type are also provided.

In Section 2, after introducing some necessary definitions, we give two combinatorial interpretations for the coefficient $a_{n, k}^{(i)}$ in $\operatorname{JS}_{n}^{k}(z)(0 \leqslant i \leqslant n-k)$, and explicitly construct a bijection between the two models. In Section 3, we give a combinatorial interpretation for the coefficient $b_{n, k}^{(i)}$ in $\mathrm{js}_{n}^{k}(z)(0 \leqslant i \leqslant n-k)$. In Section 4, we give the combinatorial interpretation for two sequences which are multiples of the central factorial numbers of odd indices and we also establish a simple derivation of the explicit formula of Jacobi-Stirling numbers.

\section{Jacobi-Stirling numbers of the second kind $\operatorname{JS}_{n}^{k}(z)$}

\subsection{First interpretation}

For any positive integer $n$, we define

$$
[ \pm n]_{0}:=\{0,1,-1,2,-2,3,-3, \ldots, n,-n\} .
$$

The following definition is equivalent to that given by Andrews and Littlejohn [1] in order to interpret Legendre-Stirling numbers, where 0 is added to avoid empty block and also to be consistent with the model for the Jacobi-Stirling numbers of the first kind. 
Definition 1. A signed $k$-partition of $[ \pm n]_{0}$ is a set partition of $[ \pm n]_{0}$ with $k+1$ non-empty blocks $B_{0}, B_{1}, \ldots B_{k}$ with the following rules:

1. $0 \in B_{0}$ and $\forall i \in[n],\{i,-i\} \not \subset B_{0}$,

2. $\forall j \in[k]$ and $\forall i \in[n]$, we have $\{i,-i\} \subset B_{j} \Longleftrightarrow i=\min B_{j} \cap[n]$.

For example, the partition $\pi=\left\{\{2,-5\}_{0},\{ \pm 1,-2\},\{ \pm 3\},\{ \pm 4,5\}\right\}$ is a signed 3 partition of $[ \pm 5]_{0}$, with $\{2,-5\}_{0}:=\{0,2,-5\}$ being the zero-block.

Theorem 2. For any positive integers $n$ and $k$, the integer $a_{n, k}^{(i)}(0 \leqslant i \leqslant n-k)$ is the number of signed $k$-partitions of $[ \pm n]_{0}$ such that the zero-block contains $i$ signed entries.

Proof. Let $\mathcal{A}_{n, k}^{(i)}$ be the set of signed $k$-partitions of $[ \pm n]_{0}$ such that the zero-block contains $i$ signed entries and $\tilde{a}_{n, k}^{(i)}=\left|\mathcal{A}_{n, k}^{(i)}\right|$. By convention $\tilde{a}_{0,0}^{(0)}=1$. Clearly $\tilde{a}_{1,1}^{(0)}=1$ and for $\tilde{a}_{n, k}^{(i)} \neq 0$ we must have $n \geqslant k \geqslant 1$ and $0 \leqslant i \leqslant n-k$. We divide $\mathcal{A}_{n, k}^{(i)}$ into four parts:

(i) the signed $k$-partitions of $[ \pm n]_{0}$ with $\{-n, n\}$ as a block. Clearly, the number of such partitions is $\tilde{a}_{n-1, k-1}^{(i)}$.

(ii) the signed $k$-partitions of $[ \pm n]_{0}$ with $n$ in the zero-block. We can construct such partitions by first constructing a signed $k$-partition of $[ \pm(n-1)]_{0}$ with $i$ signed entries in the zero block and then insert $n$ into the zero block and $-n$ into one of the $k$ other blocks; so there are $k \tilde{a}_{n-1, k}^{(i)}$ such partitions.

(iii) the signed $k$-partitions of $[ \pm n]_{0}$ with $-n$ in the zero-block. We can construct such partitions by first constructing a signed $k$-partition of $[ \pm(n-1)]_{0}$ with $i-1$ signed entries in the zero-block, and then placing $n$ into one of the $k$ non-empty blocks, so there are $k \tilde{a}_{n-1, k}^{(i-1)}$ possibilities.

(iv) the signed $k$-partitions of $[ \pm n]_{0}$ where neither $n$ nor $-n$ appears in the zero-block and $\{-n, n\}$ is not a block. We can construct such partitions by first choosing a signed $k$-partition of $[ \pm(n-1)]_{0}$ with $i$ signed entries in the zero block, and then placing $n$ and $-n$ into two different non-zero blocks, so there are $k(k-1) \tilde{a}_{n-1, k}^{(i)}$ possibilities.

Summing up we get the following equation:

$$
\tilde{a}_{n, k}^{(i)}=\tilde{a}_{n-1, k-1}^{(i)}+k \tilde{a}_{n-1, k}^{(i-1)}+k^{2} \tilde{a}_{n-1, k}^{(i)} .
$$

By (1.4), it is easy to see that $a_{n, k}^{(i)}$ satisfies the same recurrence and initial conditions as $\tilde{a}_{n, k}^{(i)}$, so they agree.

Since $\operatorname{LS}(n, k)=\sum_{i=0}^{n-k} a_{n, k}^{(i)}$, Theorem 2 implies immediately the following result of Andrews and Littlejohn [1]. 
Table 3: The first values of $\operatorname{JS}_{n}^{k}(z)$ in the basis $\left\{(z+1)^{i}\right\}_{i=0, \ldots, n-k}$

\begin{tabular}{c|ccccc}
$k \backslash n$ & 1 & 2 & 3 & 4 & 5 \\
\hline 1 & 1 & $(z+1)$ & $(z+1)^{2}$ & $(z+1)^{3}$ & $(z+1)^{4}$ \\
2 & & 1 & $2+3(z+1)$ & $4+10(z+1)+7(z+1)^{2}$ & $8+28(z+1)+34(z+1)^{2}+15(z+1)^{3}$ \\
3 & & & 1 & $8+6(z+1)$ & $52+70(z+1)+25(z+1)^{2}$ \\
4 & & & & 1 & $20+10(z+1)$ \\
5 & & & & & 1
\end{tabular}

Corollary 1. The integer $\operatorname{LS}(n, k)$ is the number of signed $k$-partitions of $[ \pm n]_{0}$.

By Theorems 1 and 2, we derive that the integer $S(n, k)$ is the number of signed $k$ partitions of $[ \pm n]_{0}$ such that the zero-block contains $n-k$ signed entries. By definition, in this case, there is no positive entry in the zero-block. By deleting the signed entries in the remaining $k$ blocks, we recover then the following known interpretation for the Stirling number of the second kind.

Corollary 2. The integer $S(n, k)$ is the number of partitions of $[n]$ in $k$ blocks.

For a partition $\pi=\left\{B_{1}, B_{2}, \ldots, B_{k}\right\}$ of $[n]$ in $k$ blocks, denote by $\min \pi$ the set of minima of blocks

$$
\min \pi=\left\{\min \left(B_{1}\right), \ldots, \min \left(B_{k}\right)\right\} .
$$

The following partition version of Dumont's interpretation for the central factorial number of even indices can be found in [6, Chap. 3].

Corollary 3. The integer $U(n, k)$ is the number of ordered pairs $\left(\pi_{1}, \pi_{2}\right)$ of partitions of $[n]$ in $k$ blocks such that $\min \left(\pi_{1}\right)=\min \left(\pi_{2}\right)$.

Proof. As $U(n, k)=a_{n, k}^{(0)}$, by Theorem 2, the integer $U(n, k)$ counts the number of signed $k$-partitions of $[ \pm n]_{0}$ such that the zero-block doesn't contain any signed entry. For any such a signed $k$-partition $\pi$, we apply the following algorithm: (i) move each positive entry $j$ of the zero-block into the block containing $-j$ to obtain a signed $k$-partition $\pi^{\prime}=\left\{\{0\}, B_{1}, \ldots, B_{k}\right\}$, (ii) $\pi_{1}$ is obtained by deleting the negative entries in each block $B_{i}$ of $\pi^{\prime}$, and $\pi_{2}$ is obtained by deleting the positive entries and taking the opposite values of signed entries in each block of $\pi^{\prime}$. For example, if $\pi=\left\{\{3\}_{0},\{ \pm 1,-3,4\},\{ \pm 2,-4\}\right\}$ is the signed 2-partition of $[ \pm 4]_{0}$, the corresponding ordered pair of partitions is $\left(\pi_{1}, \pi_{2}\right)$ with $\pi_{1}=\{\{1,3,4\},\{2\}\}$ and $\pi_{2}=\{\{1,3\},\{2,4\}\}$.

The following result shows that the coefficients in the expansion of the Jacobi-Stirling numbers $\mathrm{JS}_{n}^{k}(z)$ in the basis $\left\{(z+1)^{i}\right\}_{i=0, \ldots, n-k}$ are also interesting.

Theorem 3. Let

$$
\mathrm{JS}_{n}^{k}(z)=d_{n, k}^{(0)}+d_{n, k}^{(1)}(z+1)+\cdots+d_{n, k}^{(n-k)}(z+1)^{n-k} .
$$

Then the coefficient $d_{n, k}^{(i)}$ is a positive integer, which counts the number of signed $k$ partitions of $[ \pm n]_{0}$ such that the zero-block contains only zero and $i$ negative values. 
Proof. We derive from (1.4) that the coefficients $d_{n, k}^{(i)}$ verify the following recurrence relation:

$$
d_{n, k}^{(i)}=d_{n-1, k-1}^{(i)}+k d_{n-1, k}^{(i-1)}+k(k-1) d_{n-1, k}^{(i)} .
$$

As for the $a_{n, k}^{(i)}$, we can prove the result by a similar argument as in proof of Theorem 2 .

Corollary 4. The integer $J_{n}^{k}(-1)=d_{n, k}^{(0)}$ is the number of signed $k$-partitions of $[ \pm n]_{0}$ with $\{0\}$ as zero-block.

Remark 1. A priori, it was not obvious that $J_{n}^{k}(-1)=\sum_{i=i}^{n-k}(-1)^{i} a_{n, k}^{(i)}$ was positive.

From Theorem 1 and (2.2), we derive the following relations :

$$
a_{n, k}^{(i)}=\sum_{j=i}^{n-k}\left(\begin{array}{l}
j \\
i
\end{array}\right) d_{n, k}^{(j)}, \quad U(n, k)=\sum_{j=i}^{n-k} d_{n, k}^{(j)}, \quad \operatorname{LS}(n, k)=\sum_{j=i}^{n-k} 2^{j} d_{n, k}^{(j)} .
$$

We can give combinatorial interpretations for these formulas. For example, for the first one, we can split the set $\mathcal{A}_{n, k}^{(i)}$ by counting the total number $j$ of elements in the zero-block $(1 \leqslant j \leqslant n-k)$. Then to construct such an element, we first take a signed $k$-partition of $[ \pm n]_{0}$ with no positive values in the zero-block, so there are $d_{n, k}^{(j)}$ possibilities, and then we choose the $j-i$ numbers that are positive among the $j$ possibilities in the zero-block. Similar proofs can be easily described for the two other formulas.

\subsection{Second interpretation}

We now propose a second model for the coefficient $a_{n, k}^{(i)}$, inspired by Foata and Schützenberger [7] and Dumont [3]. Let $\mathcal{S}_{n}$ be the set of permutations of $[n]$. In the rest of this paper, we identify any permutation $\sigma$ in $\mathcal{S}_{n}$ with its diagram $\mathcal{D}(\sigma)=\{(i, \sigma(i)): i \in[n]\}$.

For any finite set $X$, we denote by $|X|$ its cardinality. If $\alpha=(i, j) \in[n] \times[n]$, we define $\operatorname{pr}_{x}(\alpha)=i$ and $\operatorname{pr}_{y}(\alpha)=j$ to be its $x$ and $y$ projections. For any subset $Q$ of $[n] \times[n]$, we define the $x$ and $y$ projections by

$$
\operatorname{pr}_{x}(Q)=\left\{\operatorname{pr}_{x}(\alpha): \alpha \in Q\right\}, \quad \operatorname{pr}_{y}(Q)=\left\{\operatorname{pr}_{y}(\alpha): \alpha \in Q\right\}
$$

and the supdiagonal and subdiagonal parts by

$$
Q^{+}=\{(i, j) \in Q: i \leqslant j\}, \quad Q^{-}=\{(i, j) \in Q: i \geqslant j\} .
$$

Definition 2. A simply hooked $k$-quasi-permutation of $[n]$ is a subset $Q$ of $[n] \times[n]$ such that

i) $Q \subset \mathcal{D}(\sigma)$ for some permutation $\sigma$ of $[n]$,

ii) $|Q|=n-k$ and $\operatorname{pr}_{x}\left(Q^{-}\right) \cap \operatorname{pr}_{y}\left(Q^{+}\right)=\emptyset$. 
Figure 1: The diagonal hook $H_{4}$ and simply hooked quasi-permutation of [6].

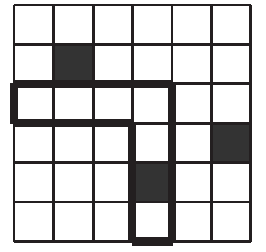

Figure 2: An ordered pair of simply hooked quasi-permutations in $\mathcal{C}_{10,3}^{(3)}$
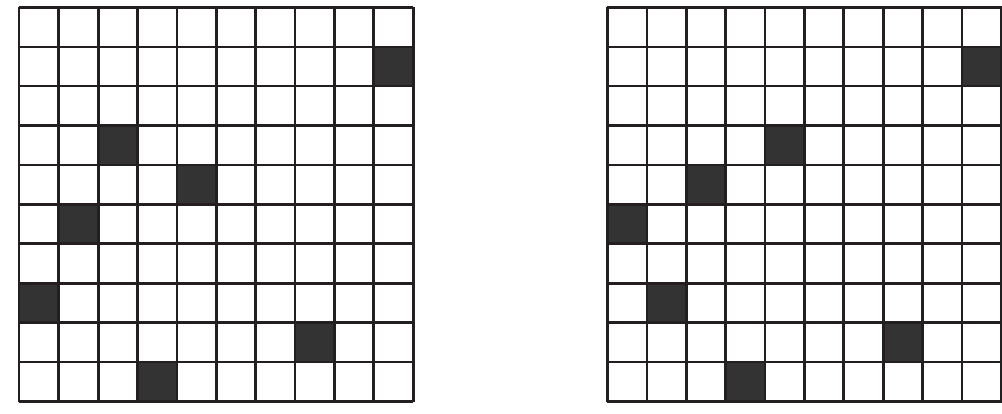

A simply hooked $k$-quasi-permutation $Q$ of $[n]$ can be depicted by darkening the $n-k$ corresponding boxes of $Q$ in the $n \times n$ square tableau. Conversely, if we define the diagonal hook $H_{i}:=\{(i, j): i \leqslant j\} \cup\{(j, i): i \leqslant j\}(1 \leqslant i \leqslant n)$, then a black subset of the $n \times n$ square tableau represents a simply hooked quasi-permutation if there is no black box on the main diagonal and at most one black box in each row, in each column and in each diagonal hook. An example is given in Figure 1.

Theorem 4. The integer $a_{n, k}^{(i)}(1 \leqslant i \leqslant n-k)$ is the number of ordered pairs $\left(Q_{1}, Q_{2}\right)$ of simply hooked $k$-quasi-permutations of $[n]$ satisfying the following conditions:

$$
Q_{1}^{-}=Q_{2}^{-}, \quad\left|Q_{1}^{-}\right|=\left|Q_{2}^{-}\right|=i \quad \text { and } \quad \operatorname{pr}_{y}\left(Q_{1}\right)=\operatorname{pr}_{y}\left(Q_{2}\right)
$$

Proof. Let $\mathcal{C}_{n, k}^{(i)}$ be the set of ordered pairs $\left(Q_{1}, Q_{2}\right)$ of simply hooked $k$-quasi-permutations of $[n]$ verifying $(2.5)$, and let $c_{n, k}^{(i)}=\left|\mathcal{C}_{n, k}^{(i)}\right|$.

For example, the ordered pair $\left(Q_{1}, Q_{2}\right)$ with

$$
\begin{aligned}
& Q_{1}=\{(1,3),(2,5),(3,7),(4,1),(5,6),(8,2),(10,9)\}, \\
& Q_{2}=\{(1,5),(2,3),(3,6),(4,1),(5,7),(8,2),(10,9)\},
\end{aligned}
$$

is an element of $\mathcal{C}_{10,3}^{(3)}$. A graphical representation is given in Figure 2.

We divide the set $\mathcal{C}_{n, k}^{(i)}$ into three parts:

- the ordered pairs $\left(Q_{1}, Q_{2}\right)$ such that the $n$-th rows and $n$-th columns of $Q_{1}$ and $Q_{2}$ are empty. Clearly, there are $c_{n-1, k-1}^{(i)}$ such elements. 
- the ordered pairs $\left(Q_{1}, Q_{2}\right)$ such that the $n$-th columns of $Q_{1}$ and $Q_{2}$ are not empty. We can first construct an ordered pair $\left(Q_{1}^{\prime}, Q_{2}^{\prime}\right)$ of $\mathcal{C}_{n-1, k}^{(i-1)}$ and then choose a box in the same position of the $n$-th column of both simply hooked quasi-permutations, there are $n-1-(n-k-1)=k$ positions available. So there are $k c_{n-1, k}^{(i-1)}$ such elements.

- the ordered pairs $\left(Q_{1}, Q_{2}\right)$ such that the $n$-th rows of $Q_{1}$ and $Q_{2}$ are not empty. We can first construct an ordered pair $\left(Q_{1}^{\prime}, Q_{2}^{\prime}\right)$ of $\mathcal{C}_{n-1, k}^{(i)}$ and then add a black box in the top of both simply hooked quasi-permutations, the box can be placed on any of the $n-1-(n-k-1)=k$ positions whose columns are empty. So there are $k^{2} c_{n-1, k}^{(i)}$ such elements.

In conclusion, we obtain the recurrence

$$
c_{n, k}^{(i)}=c_{n-1, k-1}^{(i)}+k c_{n-1, k}^{(i-1)}+k^{2} c_{n-1, k}^{(i)} .
$$

By (1.4), we see that $a_{n, k}^{(i)}$ satisfies the same recurrence relation and the initial conditions as $c_{n, k}^{(i)}$, so they agree.

Remark 2. In the first model, we don't have a direct interpretation for the integer $k^{2}$ in (2.1) because it results from after the simplification $k+k(k-1)=k^{2}$. While in the second one, we can see what the coefficient $k^{2}$ counts in $(2.7)$.

Definition 3. A supdiagonal (resp. subdiagonal) quasi-permutation of $[n]$ is a simply hooked quasi-permutation $Q$ of $[n]$ with $Q^{-}=\emptyset\left(\operatorname{resp} . Q^{+}=\emptyset\right)$.

From Theorems 1 and 4, we recover Dumont's combinatorial interpretation for the central factorial numbers of the second kind [3], and Riordan's interpretation for the Stirling numbers of the second kind (see [7, Prop. 2.7]).

Corollary 5. The integer $U(n, k)$ is the number of ordered pairs $\left(Q_{1}, Q_{2}\right)$ of supdiagonal $k$-quasi-permutations of $[n]$ such that $\operatorname{pr}_{y}\left(Q_{1}\right)=p r_{y}\left(Q_{2}\right)$.

Corollary 6. The integer $S(n, k)$ is the number of subdiagonal (resp. supdiagonal) $k$ quasi-permutations of $[n]$.

Remark 3. To recover the classical interpretation of $S(n, k)$ in Corollary 2, we can apply a simple bijection, say $\varphi$, in [7, Prop. 3]. Starting from a $k$-partition $\pi=\left\{B_{1}, \ldots, B_{k}\right\}$ of $[n]$, for each non-singleton block $B_{i}=\left\{p_{1}, p_{2}, \ldots, p_{n_{i}}\right\}$ with $n_{i} \geqslant 2$ elements $p_{1}<p_{2}<$ $\ldots<p_{n_{i}}$, we associate the subdiagonal quasi-permutation

$$
Q_{i}=\left\{\left(p_{n_{i}}, p_{n_{i-1}}\right),\left(p_{n_{i-1}}, p_{n_{i-2}}\right), \ldots,\left(p_{2}, p_{1}\right)\right\}
$$

with $n_{i}-1$ elements of $[n] \times[n]$. Clearly, the union of all such $Q_{i}^{\prime} s$ is a subdiagonal quasi-permutation of cardinality $n-k$. An example of the map $\varphi$ is given in Figure 3 . 
Figure 3: The subdiagonal quasi-permutation corresponding to a partition via the map $\varphi$

$$
\pi=\{\{1,4,6\},\{2,5\},\{3\}\} \longrightarrow
$$

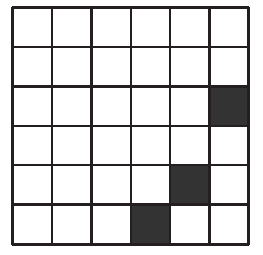

Finally, we derive from Theorem 4 and (1.14) a new combinatorial interpretation for the Legendre-Stirling numbers of the second kind. The correspondence between the two models will be established in the next subsection.

Corollary 7. The integer $\operatorname{LS}(n, k)$ is the number of ordered pairs $\left(Q_{1}, Q_{2}\right)$ of simply hooked $k$-quasi-permutations of $[n]$ such that $p r_{y}\left(Q_{1}\right)=p r_{y}\left(Q_{2}\right)$.

Remark 4. We haven't found an interpretation neither for the numbers $d_{n, k}^{(i)}$ in (2.2), nor for the formulas expressed in (2.4), in terms of simply hooked quasi-permutations.

\subsection{The link between the two models}

We introduce a third interpretation which permits to make the connection easier between the two previous models. Let $\Pi_{n, k}$ be the set of partitions of $[n]$ in $k$ non-empty blocks.

Definition 4. Let $\mathcal{B}_{n, k}^{(i)}$ be the set of triples $\left(\pi_{1}, \pi_{2}, \pi_{3}\right)$ in $\Pi_{n, k+i} \times \Pi_{n, k+i} \times \Pi_{n, n-i}$ such that:

i) $\min \left(\pi_{1}\right)=\min \left(\pi_{2}\right)$ and $\operatorname{Sing}\left(\pi_{1}\right)=\operatorname{Sing}\left(\pi_{2}\right)$,

ii) $\min \left(\pi_{1}\right) \cup \operatorname{Sing}\left(\pi_{3}\right)=\operatorname{Sing}\left(\pi_{1}\right) \cup \min \left(\pi_{3}\right)=[n]$,

where $\operatorname{Sing}(\pi)$ denotes the set of singletons in $\pi$.

We will need the following result.

Lemma 5. For $\left(\pi_{1}, \pi_{2}, \pi_{3}\right) \in \mathcal{B}_{n, k}^{(i)}$, we have:

i) $\left|\min \left(\pi_{1}\right) \cap \min \left(\pi_{3}\right)\right|=k$,

ii) $\left|\operatorname{Sing}\left(\pi_{1}\right) \backslash \min \left(\pi_{3}\right)\right|=i$,

iii) $\left|\operatorname{Sing}\left(\pi_{3}\right) \backslash \min \left(\pi_{1}\right)\right|=n-k-i$.

Proof. By definition, we have $\left|\min \left(\pi_{1}\right)\right|=k+i$ and $\left|\min \left(\pi_{3}\right)\right|=n-i$. Since $\min \left(\pi_{1}\right) \cup$ $\min \left(\pi_{3}\right)=[n]$, by sieve formula, we deduce

$$
\left|\min \left(\pi_{1}\right) \cap \min \left(\pi_{3}\right)\right|=\left|\min \left(\pi_{1}\right)\right|+\left|\min \left(\pi_{3}\right)\right|-\left|\min \left(\pi_{1}\right) \cup \min \left(\pi_{3}\right)\right|=k,
$$

and

$$
\left|\operatorname{Sing}\left(\pi_{1}\right) \backslash \min \left(\pi_{3}\right)\right|=\left|\operatorname{Sing}\left(\pi_{1}\right)\right|-\left|\operatorname{Sing}\left(\pi_{1}\right) \cap \min \left(\pi_{3}\right)\right|=n-\left|\min \left(\pi_{3}\right)\right|=i .
$$

In the same way, we obtain iii). 
Theorem 6. There is a bijection between $\mathcal{A}_{n, k}^{(i)}$ and $\mathcal{B}_{n, k}^{(i)}$.

Proof. Let $\pi=\left\{B_{0}, B_{1}, \ldots, B_{k}\right\}$ be a signed $k$-partition in $\mathcal{A}_{n, k}^{(i)}$. We construct the triple $\left(\pi_{1}, \pi_{2}, \pi_{3}\right)$ of partitions by the following algorithm.

$\pi_{1}, \pi_{2}$ : $\quad$ Let $\pi^{\prime}=\left\{B_{0}^{\prime}, B_{1}^{\prime}, \ldots, B_{k}^{\prime}\right\}$ be the partition obtained by exchanging all $j$ and $-j$ in $\pi$ if $j \in B_{0}$ (resp. $j \in[n]$ ).

- Let $\pi^{\prime \prime}=\left\{B_{0}^{\prime \prime}, B_{1}^{\prime \prime}, \ldots, B_{k}^{\prime \prime}\right\}$ be the partition obtained by removing all the negative values in $\pi^{\prime}$.

- Define $\pi_{1}$ (resp. $\pi_{2}$ ) to be the partition obtained by splitting the $i$ positive elements in $B_{0}^{\prime \prime}$ into $i$ singletons and deleting 0 in $\pi^{\prime \prime}$.

The resulting partitions $\pi_{1}$ and $\pi_{2}$ are clearly elements of $\Pi_{n, k+i}$ and satisfy $\min \left(\pi_{1}\right)=\min \left(\pi_{2}\right)$ and $\operatorname{Sing}\left(\pi_{1}\right)=\operatorname{Sing}\left(\pi_{2}\right)$.

$\pi_{3}$ : $\quad$ For all $p \in[n] \backslash \min \pi$ such that $B_{0} \cap\{ \pm p\}=\emptyset$, move $p$ into the zero-block and obtain the partition $\pi^{\prime}=\left\{B_{0}^{\prime}, B_{1}^{\prime}, \ldots, B_{k}^{\prime}\right\}$. So there are $n-k-i$ positive entries in the new $B_{0}^{\prime}$.

- Let $\pi^{\prime \prime}=\left\{B_{0}^{\prime \prime}, B_{1}^{\prime \prime}, \ldots, B_{k}^{\prime \prime}\right\}$ be the partition obtained by removing all the negative values in $\pi^{\prime}$.

- Define $\pi_{3}$ to be the partition obtained by splitting the $n-k-i$ positive elements in $B_{0}^{\prime \prime}$ into $n-k-i$ singletons and deleting 0 in $\pi^{\prime \prime}$.

The resulting partition $\pi_{3}$ is an element of $\Pi_{n, n-i}$.

For any $p \in[n] \backslash \min \left(\pi_{1}\right)$, if $p \notin B_{0}$ then $B_{0} \cap\{ \pm p\} \neq \emptyset$, by definition $p$ will be moved in the zero-block, otherwise $p$ is already in the zero-block. Thus, the elements that are not in $\min \left(\pi_{1}\right)$ become singletons in $\pi_{3}$. Hence $\min \left(\pi_{1}\right) \cup \operatorname{Sing}\left(\pi_{3}\right)=[n]$. Similarly we have $\operatorname{Sing}\left(\pi_{1}\right) \cup \min \left(\pi_{3}\right)=[n]$.

For example, for the signed 3-partition of $[ \pm 10]_{0}$ :

$$
\pi=\left\{\{-4,6,7,-8,-10\}_{0},\{ \pm 1,3,4,-5,-7\},\{ \pm 2,-3,5,-6,8\},\{ \pm 9,10\}\right\},
$$

the corresponding triple is $\left(\pi_{1}, \pi_{2}, \pi_{3}\right) \in \Pi_{10,6} \times \Pi_{10,6} \times \Pi_{10,7}$ with :

$$
\begin{gathered}
\pi_{1}=\{\{1,3,7\},\{2,5,6\},\{4\},\{8\},\{9\},\{10\}\}, \\
\pi_{2}=\{\{1,5,7\},\{2,3,6\},\{4\},\{8\},\{9\},\{10\}\}, \\
\pi_{3}=\{\{1,4\},\{2,8\},\{3\},\{5\},\{6\},\{7\},\{9,10\}\} .
\end{gathered}
$$

Conversely, for any $\left(\pi_{1}, \pi_{2}, \pi_{3}\right) \in \mathcal{B}_{n, k}^{(i)}$, we construct $\pi=\left\{B_{0}, B_{1}, \ldots, B_{k}\right\} \in \mathcal{A}_{n, k}^{(i)}$ with the following procedure:

- Use the $k$ elements of $\min \left(\pi_{1}\right) \cap \min \left(\pi_{3}\right)$, say $p_{1}, \ldots, p_{k}$ and 0 to create $k+1$ blocks:

$$
B_{0}=\{\ldots\}_{0}, B_{1}=\left\{ \pm p_{1}, \ldots\right\}, \ldots, B_{k}=\left\{ \pm p_{k} \ldots\right\}
$$

where "..." means that the blocks are not completed. For instance, for the triple $\left(\pi_{1}, \pi_{2}, \pi_{3}\right)$ in $(2.9)$, we create four blocks: $\{0, \ldots\},\{ \pm 1, \ldots\},\{ \pm 2, \ldots\}$ and $\{ \pm 9, \ldots\}$. 
- For each element $x_{j}$ of $[n] \backslash \min \left(\pi_{3}\right)(1 \leqslant j \leqslant i)$, suppose that $x_{j}$ appears in a non-singleton block $C_{j}$ of $\pi_{3}$. Then put $-x_{j}$ into the zero-block $B_{0}$ and $x_{j}$ into the block in (2.10) that contains $\min \left(C_{j}\right)$. Note that we must show that $\min \left(C_{j}\right) \in$ $\min \left(\pi_{1}\right) \cap \min \left(\pi_{3}\right)$ to warrant the existence of such a block in (2.10). Indeed, if $\min \left(C_{j}\right) \notin \min \left(\pi_{1}\right)$, then, by Definition 4 , we would have $\min \left(C_{j}\right) \in \operatorname{Sing}\left(\pi_{3}\right)$. For the current example, we place the number 4 in the block that contains 1 .

- For each element $y_{j}$ of $[n] \backslash \min \left(\pi_{2}\right)(1 \leqslant j \leqslant n-k-i)$, suppose that $y_{j}$ appears in a non-singleton block $D_{j}$ (resp. $\left.E_{j}\right)$ of $\pi_{2}\left(\right.$ resp. $\left.\pi_{1}\right)$. Then put $-p_{j}$ into the block in (2.10) that contains $\min \left(D_{j}\right)$ and put $p_{j}$ into the block in (2.10) that contains $\min \left(E_{j}\right)$ if this block dosn't contains $-p_{j}$, into the zero-block $B_{0}$ otherwise. For the current example, we place the number -3 in the block that contains 2 . and 5 in the block that contains 2 , and 6 in the zero-block because the block that contains 2 already has -6 .

Since $\varphi$ described in Remark 3 maps each partition to a subdiagonal quasi-permutation, for every triple $\left(\pi_{1}, \pi_{2}, \pi_{2}\right)$ of partitions satisfying the conditions of Theorem 6 , we can associate a triple $\left(P_{1}, P_{2}, P_{3}\right)=\left(\varphi\left(\pi_{1}\right), \varphi\left(\pi_{2}\right), \varphi\left(\pi_{3}\right)\right)$ of subdiagonal quasi-permutations. If $\overline{P_{i}}$ denotes the supdiagonal quasi-permutation obtained from $P_{i}$ exchanging the $x$ and $y$ coordonates, then $\left(Q_{1}, Q_{2}\right)=\left(\overline{P_{1}} \cup P_{3}, \overline{P_{2}} \cup P_{3}\right)$ is an ordered pair of simply hooked quasipermutations satisfying the conditions of Theorem 4 . Thus, we obtain a bijection between the signed $k$-partitions and the ordered pairs of simply hooked quasi-permutations.

For example, for the signed 3-partition $\pi$ in (2.8), the corresponding ordered pair of simply hooked quasi-permutations $\left(Q_{1}, Q_{2}\right)$ is then given by $(2.6)$ (cf. Figure 2).

\section{Jacobi-Stirling numbers of the first kind $j s_{n}^{k}(z)$}

For a permutation $\sigma$ of $[n]_{0}:=[n] \cup\{0\}$ (resp. $[n]$ ) and for $j \in[n]_{0}$ (resp. $[n]$ ), denote by $\operatorname{Orb}_{\sigma}(j)=\left\{\sigma^{\ell}(j): \ell \geqslant 1\right\}$ the orbit of $j$ and $\min (\sigma)$ the set of its cyclic minima, i.e.,

$$
\min (\sigma)=\left\{j \in[n]: j=\min \left(\operatorname{Orb}_{\sigma}(j) \cap[n]\right)\right\} .
$$

Definition 5. Given a word $w=w(1) \ldots w(\ell)$ on the finite alphabet $[n]$, a letter $w(j)$ is a record of $w$ if $w(k)>w(j)$ for every $k \in\{1, \ldots, j-1\}$. We define $\operatorname{rec}(w)$ to be the number of records of $w$ and $\operatorname{rec}_{0}(w)=\operatorname{rec}(w)-1$.

For example, if $w=\mathbf{5 7 4 8 6 2 3 1 9}$, then the records are $5,4,2,1$. Hence $\operatorname{rec}(w)=4$.

Theorem 7. The integer $b_{n, k}^{(i)}$ is the number of ordered pairs $(\sigma, \tau)$ such that $\sigma($ resp. $\tau)$ is a permutation of $[n]_{0}$ (resp. $\left.[n]\right)$ with $k$ cycles, satisfying

i) $1 \in \operatorname{Orb}_{\sigma}(0)$,

ii) $\min \sigma=\min \tau$,

iii) $\operatorname{rec}_{0}(w)=i$, where $w=\sigma(0) \sigma^{2}(0) \ldots \sigma^{l}(0)$ with $\sigma^{l+1}(0)=0$. 
Proof. Let $\mathcal{E}_{n, k}^{(i)}$ be the set of ordered pairs $(\sigma, \tau)$ satisfying the conditions of Theorem 7 and $e_{n, k}^{(i)}=\left|\mathcal{E}_{n, k}^{(i)}\right|$. We divide $\mathcal{E}_{n, k}^{(i)}$ into three parts:

(i) the ordered pairs $(\sigma, \tau)$ such that $\sigma^{-1}(n)=n$. Then $n$ forms a cycle in both $\sigma$ and $\tau$ and there are clearly $e_{n-1, k-1}^{(i)}$ possibilities.

(ii) the ordered pairs $(\sigma, \tau)$ such that $\sigma^{-1}(n)=0$. We can construct such ordered pairs by first choosing an ordered pair $\left(\sigma^{\prime}, \tau^{\prime}\right)$ in $\mathcal{E}_{n-1, k}^{(i-1)}$ and then inserting $n$ in $\sigma^{\prime}$ as image of 0 (resp. in $\tau^{\prime}$ ). Clearly, there are $(n-1) e_{n-1, k}^{(i-1)}$ possibilities.

(iii) the ordered pairs $(\sigma, \tau)$ such that $\sigma^{-1}(n) \notin\{0, n\}$. We can construct such ordered pairs by first choosing an ordered pair $\left(\sigma^{\prime}, \tau^{\prime}\right)$ in $\mathcal{E}_{n-1, k}^{(i)}$ and then inserting $n$ in $\sigma^{\prime}$ (resp. in $\tau^{\prime}$ ). Clearly, there are $(n-1)^{2} e_{n-1, k}^{(i)}$ possibilities.

Summing up, we get the following equation:

$$
e_{n, k}^{(i)}=e_{n-1, k-1}^{(i)}+(n-1) e_{n-1, k}^{(i-1)}+(n-1)^{2} e_{n-1, k}^{(i)} .
$$

By (1.5), it is easy to see that the coefficients $b_{n, k}^{(i)}$ satisfy the same recurrence.

We show now how to derive from Theorems 1 and 7 the combinatorial interpretations for the numbers $|\operatorname{ls}(n, k)|,|s(n, k)|$ and $|u(n, k)|$.

Corollary 8. The integer $|\operatorname{ls}(n, k)|$ is the number of ordered pairs $(\sigma, \tau)$ such that $\sigma$ (resp. $\tau$ ) is a permutation of $[n]_{0}$ (resp. $[n]$ ) with $k$ cycles, satisfying $1 \in \operatorname{Orb}_{\sigma}(0)$ and $\min \sigma=\min \tau$.

Corollary 9. The integer $|s(n, k)|$ is the number of permutations of $[n]$ with $k$ cycles.

Proof. By Theorem 7, the integer $|s(n, k)|$ is the number of ordered pairs $(\sigma, \tau)$ in $\mathcal{E}_{n, k}^{(n-k)}$. Since $\sigma$ and $\tau$ both have $k$ cycles with same cyclic minima, the permutation $\sigma$ is completely determinated by $\tau$ because $\operatorname{Orb}_{\sigma}(1)$ is the only non singleton cycle, of cardinality $n-k+2$, so the $n-k$ elements different from 0 and 1 are exactly the elements of $[n] \backslash \min \tau$ arranged in decreasing order in the word $w=\sigma(0) \sigma^{2}(0) \ldots 1$ with $\sigma(1)=0$.

The following result is the analogue interpretation to Corollary 3 for the central factorial numbers of the first kind. This analogy is comparable with that of Stirling numbers of the first kind $|s(n, k)|$ versus the Stirling numbers of the second kind $|S(n, k)|$.

Corollary 10. The integer $|u(n, k)|$ is the number of ordered pairs $(\sigma, \tau) \in \mathcal{S}_{n}^{2}$ with $k$ cycles, $\operatorname{such}$ that $\min (\sigma)=\min (\tau)$.

Indeed, the integer $|u(n, k)|$ is the number of ordered pairs $(\sigma, \tau)$ in $\mathcal{E}_{n, k}^{(0)}$. Theorem 7 implies that $\sigma^{-1}(1)=0$. The result follows then by deleting the zero in $\sigma$.

Remark 5. By the substitution $i \rightarrow n+1-i$, we can derive that the number $|u(n, k)|$ is also the number of ordered pairs $(\sigma, \tau)$ in $\mathcal{S}_{n}^{2}$ with $k$ cycles, such that $\max (\sigma)=\max (\tau)$, where $\max (\sigma)$ is the set of cyclic maxima of $\sigma$, i.e.,

$$
\max (\sigma)=\left\{j \in[n]: j=\max \left(\operatorname{Orb}_{\sigma}(j)\right\} .\right.
$$


Table 4: The first values of $V(n, k)$ and $|v(n, k)|$

\begin{tabular}{|c|c|c|c|c|c|c|c|c|c|c|c|c|c|}
\hline$k \backslash n$ & 0 & 1 & 2 & 3 & 4 & 5 & $k \backslash n$ & 0 & 1 & 2 & 3 & 4 & 5 \\
\hline 0 & 1 & 1 & 1 & 1 & 1 & 1 & 0 & 1 & 1 & 9 & 225 & 11025 & 893025 \\
\hline 1 & & 1 & 10 & 91 & 820 & 7381 & 1 & & 1 & 10 & 259 & 12916 & 1057221 \\
\hline 2 & & & 1 & 35 & 966 & 24970 & 2 & & & 1 & 35 & 1974 & 172810 \\
\hline 3 & & & & 1 & 84 & 5082 & 3 & & & & 1 & 84 & 8778 \\
\hline 4 & & & & & 1 & 165 & 4 & & & & & 1 & 165 \\
\hline 5 & & & & & & 1 & 5 & & & & & & 1 \\
\hline
\end{tabular}

\section{Further results}

\subsection{Central factorial numbers of odd indices}

For all $n, k \geqslant 0$, set

$$
V(n, k)=4^{n-k} T(2 n+1,2 k+1), \quad v(n, k)=4^{n-k} t(2 n+1,2 k+1) .
$$

Note that these numbers are also integers (see Table 4). By definition, we have the following recurrence relations :

$$
\begin{aligned}
V(n, k) & =V(n-1, k-1)+(2 k+1)^{2} V(n-1, k), \\
v(n, k) & =v(n-1, k-1)-(2 n-1)^{2} v(n-1, k) .
\end{aligned}
$$

The natural question is to find a combinatorial interpretation for these numbers. We can easily find it from combinatorial theory of generating functions.

Theorem 8. The integer $V(n, k)$ is the number of partitions of $[2 n+1]$ into $2 k+1$ blocks of odd cardinality.

Proof. This follows from the known generating function (see [8, p. 214]):

$$
\sum_{n, k \geqslant 0} V(n, k) t^{k} \frac{x^{n}}{n !}=\sinh (t \sinh (x)),
$$

and the classical combinatorial theory of generating functions (see [7, Chp. 3] and [9, Chp. 5]).

To interpret the integer $|v(n, k)|$, we need to introduce the following definition.

Definition 6. A $(n, k)$-Riordan complex is a $(2 k+1)$-tuple

$$
\left(\left(B_{1}, \sigma_{1}, \tau_{1}\right), \ldots,\left(B_{2 k+1}, \sigma_{2 k+1}, \tau_{2 k+1}\right)\right)
$$

such that 
i) $\left\{B_{1}, \ldots, B_{2 k+1}\right\}$ is a partition of $[2 n+1]$ into blocks $B_{i}$ of odd cardinality;

ii) $\sigma_{i}$ and $\tau_{i}(1 \leqslant i \leqslant 2 k+1)$ are fixed point free involutions on $B_{i} \backslash \max \left(B_{i}\right)$.

Theorem 9. The integer $|v(n, k)|$ is the number of $(n, k)$-Riordan complexes.

Proof. It is known that (see [8, p. 214]):

$$
\sum_{n, k \geqslant 0}|v(n, k)| t^{k} \frac{x^{n}}{n !}=\sinh (t \arcsin (x)),
$$

and

$$
\arcsin (x)=\sum_{n \geqslant 0}((2 n-1) ! !)^{2} \frac{x^{2 n+1}}{(2 n+1) !},
$$

where $(2 n-1) ! !=(2 n-1)(2 n-3) \cdots 3 \cdot 1$. Since $(2 n-1) ! !$ is the number of involutions without fixed points on $[2 n]$ (see [2]), the integer $((2 n-1) ! !)^{2}$ is the number of ordered pairs of involutions without fixed points on $[2 n+1] \backslash\{2 n+1\}$.

Define the numbers $J(n, m)$ by:

$$
\exp \left(t \sum_{n \geqslant 1}((2 n-1) ! !)^{2} \frac{x^{2 n+1}}{(2 n+1) !}\right)=\sum_{n, m \geqslant 0} J(2 n+1, m) t^{m} \frac{x^{2 n+1}}{(2 n+1) !} .
$$

Then, by the theory of exponential generating functions (see [7, Chp. 3] and [9, Chp. 5]), the coefficient $J(2 n+1, m)$ is the number of $m$-tuples

$$
\left(B_{1}, \sigma_{1}, \tau_{1}\right), \ldots,\left(B_{m}, \sigma_{m}, \tau_{m}\right)
$$

where $\left\{B_{1}, \ldots, B_{m}\right\}$ is a partition of $[2 n+1]$ with $\left|B_{i}\right|$ odd $(1 \leqslant i \leqslant m)$, and $\sigma_{i}$ and $\tau_{i}$ are involutions without fixed points on $B_{i} \backslash \max \left(B_{i}\right)$. As $\sinh (x)=\left(e^{x}-e^{-x}\right) / 2$, we have $|v(n, m)|=J(2 n+1,2 k+1)$ if $m=2 k+1$, and $|v(n, m)|=0$ if $m$ is even.

Remark 6. From (1.9), we can easily deduce that

$$
\sum_{k=0}^{n}|v(n, k)| t^{2 k+1}=t\left(t^{2}+1\right)\left(t^{2}+3^{2}\right) \ldots\left(t^{2}+(2 n-1)^{2}\right) .
$$

It is interesting to note that a proof of the latter result is not obvious from (4.3). In the same way, proofs for Theorems 8 and 9 by using (4.1) or (4.2) are not obvious.

Example 1. There are ten (2,1)-Riordan complexes. Since the numbers $n$ and $k$ are small, the involved involutions are identical transpositions.

$$
\begin{array}{ll}
\{1\},\{(2,3), 4\},\{5\}, & \{1\},\{2\},\{(3,4), 5\}, \\
\{(1,2), 3\},\{4\},\{5\}, & \{(1,2), 5\},\{3\},\{4\}, \\
\{(1,3), 4\},\{2\},\{5\}, & \{(1,3), 5\},\{2\},\{4\}, \\
\{(1,2), 4\},\{3\},\{5\}, & \{(1,4), 5\},\{2\},\{3\}, \\
\{1\},\{(2,3), 5\},\{4\}, & \{1\},\{(2,4), 5\},\{3\},
\end{array}
$$

where $\{1\},\{(2,3), 4\},\{5\}$ means that $\pi=\{\{1\},\{2,3,4\},\{5\}\}$, and $\sigma=\tau=13245$. 


\subsection{Generating functions}

In [5], the authors made a long calculation to derive an explicit formula for the JacobiStirling numbers. Actually, we can derive an explicit formula for the Jacobi-Stirling numbers straightforwardly from the Newton interpolation formula:

$$
x^{n}=\sum_{j=0}^{n}\left(\sum_{r=0}^{j} \frac{x_{r}^{n}}{\prod_{k \neq i}\left(x_{r}-x_{k}\right)}\right) \prod_{i=0}^{j-1}\left(x-x_{i}\right) .
$$

Indeed, making the substitutions $x \rightarrow m(z+m)$ and $x_{i} \rightarrow i(z+i)$ in (4.4), we obtain

$$
(m(m+z))^{n}=\sum_{j=0}^{n} \operatorname{JS}_{n}^{j}(z)(m-j+1)_{j}(z+m)_{j},
$$

where

$$
\mathrm{JS}_{n}^{j}(z)=\sum_{r=0}^{j}(-1)^{r} \frac{[r(r+z)]^{n}}{r !(j-r) !(z+r)_{r}(z+2 r+1)_{j-r}}
$$

and $(z)_{n}=z(z+1) \ldots(z+n-1)$.

Remark 7. If we substitute $x$ by $m(m+z)+k$, we obtain [5, Theorem 4.1].

From the recurrence (1.4), we derive:

$$
\sum_{n \geqslant k} \operatorname{JS}_{n}^{k}(z) x^{n}=\frac{x}{1-k(k+z)} \sum_{n \geqslant k-1} \operatorname{JS}_{n}^{k-1}(z) x^{n}
$$

therefore,

$$
\sum_{n \geqslant k} \operatorname{JS}_{n}^{k}(z) x^{n}=\frac{x^{k}}{(1-(z+1) x)(1-2(z+2) x) \ldots(1-k(z+k) x)}
$$

\section{Acknowledgement}

This work was partially supported by the French National Research Agency through the grant ANR-08-BLAN-0243-03.

\section{References}

[1] G. E. Andrews, L. L. Littlejohn, A combinatorial interpretation of the LegendreStirling numbers, Proc. Amer. Math. Soc. 137 (2009), 2581-2590.

[2] L. Comtet, Advanced combinatorics, Boston, Dordrecht, 1974. 
[3] D. Dumont, Interprétations combinatoires des nombres de Genocchi, Duke Math. J., t. 41, 1974, p. 305-318.

[4] W. N. Everitt, L. L. Littlejohn, R. Wellman, Legendre polynomials, Legendre-Stirling numbers, and the left-definite spectral analysis of the Legendre differential expression, J. Combut. Appl. Math., 148 (2002), 213-238.

[5] W. N. Everitt, K. H. Kwon, L. L. Littlejohn, R. Wellman, G. J. Yoon, JacobiStirling numbers, Jacobi polynomials, and the left-definite analysis of the classical Jacobi differential expression, J. Combut. Appl. Math., 208 (2007), 29-56.

[6] D. Foata, G. -N. Han, Principes de combinatoire classique, Lecture notes, Strasbourg, 2000, revised 2008.

[7] D. Foata, M. P. Schützenberger, Théorie géométrique des polynômes eulériens, Lecture Notes in Math no. 138, Springer-Verlag, Berlin, 1970.

[8] J. Riordan, Combinatorial Identities, John Wiley \& Sons, Inc., 1968.

[9] R. P. Stanley, Enumerative Combinatorics, vol.2, Cambridge Studies in Advanced Mathematics, 62, 1999. 